Appl. Phys. A 53, 514-522 (1991)

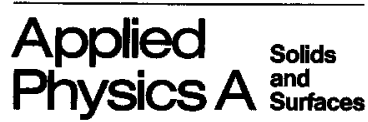

(C) Springer-Verlag 1991

\title{
High-Resolution Nonlinear Laser Spectroscopy of Exciton Relaxation in GaAs Quantum Wells
}

\author{
H. Wang and D. G. Steel \\ H. M. Randall Laboratory of Physics, The University of Michigan, Ann Arbor, MI 48109, USA
}

Received 1 May 1991/Accepted 11 September 1991

\begin{abstract}
This paper describes measurements of exciton relaxation in GaAs/AlGaAs quantum well structures based on high resolution nonlinear laser spectroscopy. The nonlinear optical measurements show that low energy excitons can be localized by monolayer disorder of the quantum well interface. We show that these excitons migrate between localization sites by phonon assisted migration, leading to spectral diffusion of the excitons. The frequency domain measurements give a direct measure of the quasi-equilibrium exciton spectral redistribution due to exciton energy relaxation, and the temperature dependence of the measured migration rates confirms recent theoretical predictions. The observed line shapes are interpreted based on solutions we obtain to modified Bloch equations which include the effects of spectral diffusion.
\end{abstract}

PACS: $71.35 .+\mathrm{z}, 42.65 .-\mathrm{k}, 78.47 .+\mathrm{p}, 78.65 . \mathrm{Fa}$

The linear and nonlinear optical properties of GaAs quantum well $(\mathrm{QW})$ structures are dominated by strong excitonic resonances near the band edge. In these materials, quasi-two-dimensional excitons are confined in the thin GaAs layer by the larger band gap of the $\mathrm{Al}_{x} \mathrm{Ga}_{1-x} \mathrm{As}$ layers. The enhancement of the electronhole correlation due to confinement leads to an increase in both the exciton oscillator strength and the exciton binding energy. The resultant appearance of well-resolved excitonic resonances even at room temperature has generated considerable interests in studies of excitonic nonlinear optical properties in QW structures [1]. It is now understood that effects of the Pauli exclusion principle including phase space filling and exchange dominate the nonlinear optical response of the exciton while effects of the Coulomb screening are significantly reduced due to the reduced dimensionality [2].

In adition to the exciton manybody interactions, the nonlinear optical property of the exciton also strongly depends on the dynamical interaction of the exciton with the surrounding crystal lattice and vacuum radiation. Relaxation of the exciton and the associated polarization (i.e., the induced coherence) due to these interactions can significantly alter the nonlinear optical response of the exciton. Measurements of the excitonic nonlinear optical response not only determine the effect of relaxation on the nonlinear optical property of the material, but also provide us a quantitative description of the exciton relaxation process. As we discuss below, details of exciton relaxation in a QW also in part reflect properties of the interface between $\mathrm{GaAs}$ and $\mathrm{AlGaAs}$.

Relaxation of excitonic excitation is characterized by decay of the exciton population about energy $E$ and decay of the optically induced polarization or coherence (often called dephasing which determines the homogeneous line width, $\left.\Gamma_{\mathrm{h}}\right)$. In an ideal crystal, excitons are described by a Bloch wave function and are free to move in the crystal. At low exciton density, decay of the excitation is expected to be predominantly due to exciton-phonon scattering along with exciton recombination.

However, in a QW the problem becomes more complicated. Non-ideal growth conditions can result in interface roughness between the GaAs well and AlGaAs barrier. Recent transport and chemical lattice imaging investigations have shown the interface of GaAs/AlGaAs multiple quantum well samples may be characterized by island-like structures with a height of one mononlayer and a lateral size of order $50 \AA$ [3]. Details of the interface roughness also depend on specific growth processes, such as interrupted or non-interrupted growth $[4,5]$, or whether GaAs is grown on AlGaAs or AlGaAs is grown on GaAs [5]. The effects of interface roughness are significant. For example, an exciton confined to a thin GaAs layer (typically $100 \AA$ ) experiences an energy shift of the 
order of several $\mathrm{meV}$ for a monolayer well-width change. As the exciton moves inside the well, it will be scattered by the random potential due to the interface roughness. As a result, low energy excitons are expected to become localized, i.e. the envelope of the wave function decays exponentially in space. In this case, the energy of the exciton also depends on the local environment leading to inhomogeneous broadening of the linear absorption spectrum.

Localized and delocalized excitons have qualitatively different relaxation properties [6]. Even at very low temperature $(<10 \mathrm{~K})$, localized excitons do not remain truly localized, insteady they can migrate among localization sites by emitting or absorbing acoustic phonons (phonon assisted migration). Phonon assisted migration was first proposed to explain the slow and non-exponential energy relaxation observed in time resolved luminescence measurements in a GaAs QW structure [6, 7]. At higher temperatures, excitons can absorb phonons of enough energy and be activated to delocalized states at higher energies. The activation process has been observed in a number of measurements such as spectral hole burning [8], resonant Rayleigh scattering [9], and resonant Raman scattering [10]. Estimations of the activation energy have suggested that the onset for the delocalized exciton in GaAs QW structures is near the absorption line center $[8,9]$. In contrast, decay of the delocalized exciton is determined by the exciton phonon scattering along the energy-momentum dispersion curve and the exciton recombination. Furthermore, delocalized excitons also experience elastic scattering from potential fluctuations, which introduces additional dephasing to the decay of the polarization.

In this paper, we discuss high resolution nonlinear optical measurements of the exciton relaxation in GaAs quantum well structures. The measurements are based on frequency domain four wave mixing (FWM) [11]. The primary objective of these measurements has been to further the physical understanding of relaxation of excitonic excitations in QW structures. Particular emphasis has also been given to the understanding of nonlinear optical measurements in semiconductors, and to how relaxation manifests itself in the frequency domain nonlinear optical response of the material. High resolution nonlinear measurements can provide detailed information on the population relaxation similar to transient nonlinear absorption measurements as well as eliminate the inhomogeneous broadening due to the interface roughness leading to a measure of the homogeneous line shape or the steady state spectral redistribution of the excitation. Since these measurements are performed in the energy space, they are particularly sensitive to relaxation processes involving energy shifts of the excitation (referred to in general as spectral diffusion) such as the exciton migration discussed above. The narrow band-width of the excitation also permits improved resolution over the usual time domain measurements. In addition, the frequency domain methods are able to observe in a single measurement time scales ranging over twelve orders of magnitude [12].

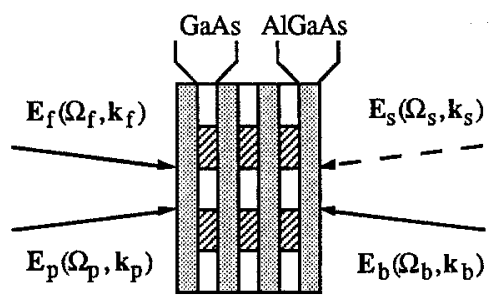

Fig. 1. A schematic representation of the experimental configuration for frequency domain backward four-wave mixing spectroscopy in GaAs/AlGaAs QW structures. The cross hatched region represents the region of optically excited excitons which are confined by the AlGaAs barriers

\section{Frequency Domain FWM Line Shapes}

The experimental configuration is based on the backward FWM geometry, which is closely related to optical phase conjugation [13], and on the use of two frequency stabilized tunable $\mathrm{cw}$ dye lasers. As shown in Fig. 1 , two counter-propagating pump beams $\mathbf{E}_{\mathrm{f}}\left(\Omega_{\mathrm{f}}, \mathbf{k}_{\mathrm{f}}\right)$ and $\mathbf{E}_{\mathrm{b}}\left(\Omega_{\mathrm{b}}, \mathbf{k}_{\mathrm{b}}\right.$ ) (f and $\mathrm{b}$ stand for forward and backward respectively) interact in the sample with a probe beam $\mathbf{E}_{\mathrm{p}}\left(\Omega_{\mathrm{p}}, \mathbf{k}_{\mathrm{p}}\right)$ with $\mathbf{E}_{\mathrm{p}} \| \mathbf{E}_{\mathrm{f}} \perp \mathbf{E}_{\mathrm{b}}$ through the resonant third order susceptibility to generate a signal beam $\mathbf{E}_{\mathrm{s}}\left(\Omega_{\mathrm{s}}, \mathbf{k}_{\mathrm{s}}\right)$ proportional to $\chi^{(3)}\left(\mathbf{E}_{\mathrm{f}} \cdot \mathbf{E}_{\mathrm{p}}^{*}\right) \mathbf{E}_{\mathrm{b}}$. Physically, $\mathbf{E}_{\mathrm{f}} \cdot \mathbf{E}_{\mathrm{p}}^{*}$ results in a spatial and temporal modulation of the exciton population, which modifies the optical response of the sample. The coherent nonlinear signal arises from scattering of the backward beam from the modulation [14]. Spectroscopic information can be obtained by studying the dependence of the nonlinear optical response on the relative frequency detuning, the electric field polarization, or the relative angle of the different input beams. For the measurements in this paper, information related to relaxation of the system is obtained by measuring the nonlinear response as a function of the frequency of any of the three input beams. The resultant line shape is designated the FWMi response where $i=f, b, p$ depending on which beam is tuned. In general, FWMp and FWMf responses provide details of population relaxation, while the FWMb response reflects a measure of the homogeneous line shape and of the spectral redistribution of the excitation as we discuss below.

To illustrate the measurement ability of the frequency domain nonlinear measurement and understand the effects of spectral diffusion on line shapes of the nonlinear optical response, we have examined the FWM response of a simple two level system undergoing spectral diffusion [15]. In this model, the excitation at an individual site can transfer to neighboring sites through an intersite interaction (such as dipole-dipole interaction). The excitation transfer is assumed to be characterized by a redistribution kernel $W\left(\omega, \omega^{\prime}\right)$ representing the rate for populations in the excited state to be transferred from a site with resonant frequency $\omega$ to sites with resonant frequency $\omega^{\prime}$. The overall spectral diffusion rate is then $\Gamma(\omega)=\int W\left(\omega, \omega^{\prime}\right) d \omega^{\prime} . W\left(\omega, \omega^{\prime}\right)$ is analogous to collision kernels that are used to describe velocity changing collisions in atomic vapor [16]. In addition, since the 
excitation transfer is likely to be associated with emission or absorption of thermal phonons, we assume that at the new site the excitation transfer does not induce a superposition state of the ground and excited state that is coherent with the input fields. Such a model has been discussed in detail elsewhere [15], and is closely related to the problem of excitation transfer between ions embedded in a crystal [17].

The interaction of the system with optical fields can be described by the following modified optical Bloch equations (MOBE) assuming a classical representation for the optical fields:

$$
\begin{aligned}
\mathrm{i} \hbar \frac{\partial}{\partial t} \varrho_{22}(\omega)= & -\left[V \varrho_{21}(\omega)-\text { c.c. }\right]-\mathrm{i} \hbar\left[\gamma_{\mathrm{sp}}+\Gamma(\omega)\right] \varrho_{22}(\omega) \\
& +\mathrm{i} \hbar \int W\left(\omega^{\prime}, \omega\right) \varrho_{22}\left(\omega^{\prime}\right) d \omega^{\prime}
\end{aligned}
$$

$$
\begin{aligned}
\mathrm{i} \hbar \frac{\partial}{\partial t} \varrho_{12}(\omega)= & V\left[\varrho_{22}(\omega)-\varrho_{11}(\omega)\right]-\hbar \omega \varrho_{12}(\omega) \\
& -\mathrm{i} \hbar\left[\gamma+\Gamma_{12}(\omega)\right] \varrho_{12}(\omega),
\end{aligned}
$$

where level $|2\rangle$ is the excited state and level $|1\rangle$ is the ground state, $\varrho_{i j}(\omega)$ is the usual population density matrix element for systems with a resonant frequency $\omega$ [18], $\Gamma_{12}(\omega)$ represents the dephasing rate of the system due to spectral diffusion, $V=-\boldsymbol{\mu} \cdot \mathbf{E}$ is the interaction energy with $\boldsymbol{\mu}$ the dipole moment (assumed to be real) and $\mathbf{E}=\frac{1}{2} \sum \mathbf{E}_{j} \exp \left(\mathbf{i k}_{j} \cdot \mathbf{x}-\mathrm{i} \Omega_{j} t\right)+$ c.c. summed over all applied electric fields. Equation (2) takes into consideration pure dephasing of the optically induced coherence by letting the dephasing rate $\gamma=\gamma_{\mathrm{sp}} / 2+\gamma_{\mathrm{ph}}$, where $\gamma_{\mathrm{sp}}$ is the spontaneous emission rate, and $\gamma_{\mathrm{ph}}$ is the pure dephasing rate, due for example to elastic scattering. In addition, we have also assumed that the system is quantum mechanically closed, i.e., the probability that an atom is in the ground or the excited state is unity. This leads to the condition $\varrho_{11}(\omega)+\varrho_{22}(\omega)=G(\omega)$ where $G(\omega)$ is the density of atoms at frequency $\omega$. Note that spectral diffusion due to intersite energy transfer is also accompanied by a corresponding change in location of the excitation. However, if the effective mean free path for excitation transfer is small, then the effect would be described by a spatial diffusion process. This effect can be phenomenologically included as a spatial diffusion decay term in the equation for $\varrho_{22}$, and the decay rate is given by $\Gamma_{\mathrm{d}}=4 \pi^{2} \mathrm{D} / \Lambda^{2}$ where $D$ is the diffusion coefficient and $A$ is the spatial period of the modulation.

The model discussed above may not accurately represent the physical process of exciton migration during nonlinear optical interaction, since a simple two level model does not reflect many-body nature of the excitonic nonlinear optical response. Moreover, description of the spectral diffusion process in general, and exciton migration in particular is much more complicated than a simple transfer of the excited state population [19]. Nevertheless, recent theoretical work has shown that in the limit that phase space filling dominates the exciton nonlinear optical response, the equation of motion governing the nonlinear optical process in semiconductors is nearly identical to the optical Bloch equation of an atomic system [20]. Hence, we expect predictions from the above simple two level model to reflect the qualitative features of FWM line shapes of more complicated systems, in particular excitons in QW structures, and provide a guidance for more detailed studies.

Even with the above assumptions, it is rather difficult to obtain analytical solutions for frequency domain FWM line shapes for a general distribution kernel. However, using a strong redistribution model for the spectral diffusion process we may further simplify the above equations. In this model, the spectral diffusion process is independent of the resonant frequency of the initial site, and each transfer on average leads to a complete redistribution of the excited state population [21], i.e. $W\left(\omega^{\prime}, \omega\right)=\Gamma F(\omega)$ with $F(\omega)$ satisfying $\int d \omega F(\omega)=1$ where $\Gamma$ is the overall spectral diffusion rate for the excited state. For the qualitative discussions of this work, the dephasing rate due to spectral diffusion is taken to be $\Gamma_{12}(\omega)=\Gamma / 2$ (the coupling giving rise to spectral diffusion may also lead to pure-dephasing effects which could be included in $\gamma_{\mathrm{ph}}$ ).

With these simplifications, the off diagonal matrix element to the third order in applied fields for a signal nearly counter-propagating to the probe (proportional to $\left.\left(\mathbf{E}_{\mathrm{f}} \cdot \mathbf{E}_{\mathrm{p}}^{*}\right) \mathbf{E}_{\mathrm{b}}\right)$ can be obtained [15]:

$$
\begin{aligned}
\varrho_{21}^{(3)}(\omega)= & -2\left(\frac{\mu}{2 \hbar}\right)^{3} E_{\mathrm{f}} E_{\mathrm{p}}^{*} E_{\mathrm{b}} \exp \left[\mathrm{i}\left(\mathbf{k}_{\mathrm{f}}+\mathbf{k}_{\mathrm{b}}-\mathbf{k}_{\mathrm{p}}\right) \cdot \mathbf{x}-\mathrm{i} \Omega_{\mathrm{s}} t\right] \\
& \times \frac{1}{\Omega_{\mathrm{s}}-\omega+\mathrm{i} \Gamma_{\mathrm{h}}} \cdot \frac{1}{\Delta_{\mathrm{fp}}+\mathrm{i}\left(\Gamma+\gamma_{\mathrm{sp}}\right)} \\
& \times\left\{G(\omega)\left(\frac{1}{\Omega_{\mathrm{f}}-\omega+\mathrm{i} \Gamma_{\mathrm{h}}}-\frac{1}{\Omega_{\mathrm{p}}-\omega-\mathrm{i} \Gamma_{\mathrm{h}}}\right)\right. \\
& \left.+\frac{\mathrm{i} \Gamma}{\Delta_{\mathrm{fp}}+\mathrm{i} \gamma_{\mathrm{sp}}} F(\omega)\left[A\left(\Omega_{\mathrm{f}}\right)-A^{*}\left(\Omega_{\mathrm{p}}\right)\right]\right\}
\end{aligned}
$$

where $\Gamma_{\mathrm{h}}=\gamma_{\mathrm{sp}} / 2+\Gamma / 2+\gamma_{\mathrm{ph}}$ is the total dephasing rate, $\Omega_{\mathrm{s}}=\Omega_{\mathrm{f}}+\Omega_{\mathrm{b}}-\Omega_{\mathrm{p}}$ is the signal frequency, and $\Delta_{\mathrm{ij}}=\Omega_{\mathrm{i}}-\Omega_{\mathrm{j}}$ with $\mathrm{i}, \mathrm{j}=\mathrm{f}, \mathrm{b}, \mathrm{p}, \mathrm{s} . A(\Omega)$ is given by

$A(\Omega)=\int \frac{1}{\Omega-\omega+\mathrm{i} \Gamma_{\mathrm{h}}} G(\omega) d \omega$.

The imaginary part of the $A(\Omega)$ is related to the inhomogeneous linear absorption profile of the system and the real part corresponds to the dispersion. The corresponding third order polarization can be easily obtained by integrating $\varrho_{21}^{(3)}(\omega)$ over the inhomogeneous distribution, yielding

$$
\begin{aligned}
P^{(3)}\left(\Omega_{\mathrm{s}}\right)= & -2 \mu\left(\frac{\mu}{2 \hbar}\right)^{3} E_{\mathrm{f}} E_{\mathrm{p}}^{*} E_{\mathrm{b}} \exp \left[\mathrm{i}\left(\mathbf{k}_{\mathrm{f}}+\mathbf{k}_{\mathrm{b}}-\mathbf{k}_{\mathrm{p}}\right) \cdot \mathbf{x}-\mathrm{i} \Omega_{\mathrm{s}} t\right] \\
& \times \frac{1}{\Delta_{\mathrm{fp}}+\mathrm{i}\left(\gamma_{\mathrm{sp}}+\Gamma\right)}\left\{\frac{1}{\Delta_{\mathrm{sp}}+2 \mathrm{i} \Gamma_{\mathrm{h}}}\left[A\left(\Omega_{\mathrm{s}}\right)-A^{*}\left(\Omega_{\mathrm{p}}\right)\right]\right. \\
& -\frac{1}{\Delta_{\mathrm{sf}}}\left[A\left(\Omega_{\mathrm{s}}\right)-A\left(\Omega_{\mathrm{f}}\right)\right]+\frac{\mathrm{i} \Gamma}{\Delta_{\mathrm{fp}}+\mathrm{i} \gamma_{\mathrm{sp}}} \\
& \left.\times Q\left(\Omega_{\mathrm{s}}\right)\left[A\left(\Omega_{\mathrm{f}}\right)-A^{*}\left(\Omega_{\mathrm{p}}\right)\right]\right\}+ \text { c.c. }
\end{aligned}
$$

where $Q\left(\Omega_{\mathrm{s}}\right)$ is related to the redistribution kernel by

$Q\left(\Omega_{\mathrm{s}}\right) \doteq \int \frac{1}{\Omega_{\mathrm{s}}-\omega+\mathrm{i} \Gamma_{\mathrm{h}}} F(\omega) d \omega$. 


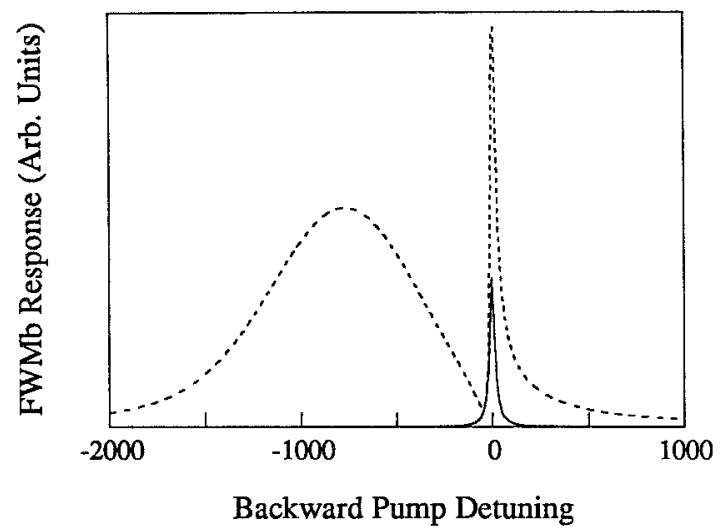

Fig. 2. The FWMb response. All the frequency and decay rates are normalized to the spontaneous emission rate. $\Gamma=15, \Delta \omega_{\text {inh }}=$ $1000, \Delta \omega_{0}=400, \Omega_{\mathrm{p}}-\omega_{\text {inh }}=-100, \omega_{0}-\omega_{\text {inh }}=-850$, where $\omega_{\text {inh }}$ and $\Delta \omega_{\text {inh }}$ are the center and half-width of the inhomogeneous distribution respectively, and $\omega_{0}$ and $\Delta \omega_{0}$ are the center and halfwidth of the redistribution kernel respectively. The backward pump detuning is measured with regard to $\Omega_{\mathrm{p}}-\Delta_{\mathrm{fp}}$. The solid line is the line shape obtained with $\Delta_{\mathrm{fp}}=\Gamma$. The dashed line is the line shape obtained with $\Delta_{\mathrm{fp}}=0$

The nonlinear optical signal is determined by a solution of the appropriate Maxwell wave equation where the nonlinear polarization is the source term. In the limit that all fields are weak and there is no depletion of any of the applied fields, the nonlinear signal is simply proportional to $\left|P^{(3)}\left(\Omega_{\mathrm{s}}\right)\right|^{2}$.

Equation (5) is nearly identical to the results obtained for a two level system without spectral diffusion with the exception of the last term [22]. The resonant denominator $\left[\Delta_{\mathrm{fp}}+\mathrm{i}\left(\gamma_{\mathrm{sp}}+\Gamma\right)\right]^{-1}$ arises in the calculation of the second order diagonal matrix element. The width of the denominator measures the decay rate of the excitation modulation, and includes an additional contribution from spectral diffusion. The familiar hole burning resonant denominator associated with $2 \Gamma_{\mathrm{h}}$ results when the signal is resonant with the dipole excited by $\mathbf{E}_{\mathrm{f}} \cdot \mathbf{E}_{\mathrm{p}}^{*}$ (i.e., when $\Omega_{\mathrm{s}}=\Omega_{\mathrm{p}}$ ), and also shows an additional broadening due to the dephasing that occurs through spectral diffusion. The term inversely proportional to $A_{\mathrm{sf}}$ is not singular, but rather goes to 0 in the limit of $\Delta_{\mathrm{sf}}=0$. Contributions from this term are negligible in the limit of strong inhomogeneous broadening when the detuning $\Delta_{\mathrm{ij}}$ is small compared with the inhomogeneous width.

The last term in (5) results from spectrally diffused excitation. It is proportional to $Q\left(\Omega_{\mathrm{s}}\right)$ reflecting a measure of the redistribution kernel. Because of the resonant enhancement which occurs when the signal is resonant with the optical transition as represented by the denominator $\left(\Omega_{\mathrm{s}}-\omega+\mathrm{i} \Gamma_{\mathrm{h}}\right)^{-1}$ in (3), the dominant contribution to the nonlinear response is expected to come from systems that are resonant with the $\Omega_{\mathrm{s}}$. In the presence of spectral diffusion, the signal coming from the last term can be observed even when the signal frequency is tuned away from the frequency of the initial excitation (the frequency of the forward pump and the probe beams) and resonant with excitations transferred to energy $E=\hbar \Omega_{\mathrm{s}}$.
We first discuss the FWMb line shape. In this measurement, nearly degenerate forward pump and probe beams interfere in the sample to excite population modulation of a narrow spectral hole with a half-width $\Gamma_{\mathrm{h}}$ within the inhomogeneous profile. The FWMb line shape is obtained by measuring the nonlinear signal as a function of the frequency of the backward pump beam. This measurement is analogous to the cw hole burning measurements and also closely related to the standard two pulse photon echo measurement [23]. In hole burning measurements, a pump beam modulates the absorption in a narrow bandwidth compared to the inhomogeneous width whereas in FWM, a population grating is created. In the absence of spectral diffusion $(\Gamma=0)$, the FWMb line shape simply reflects the spectral details of the hole excited by $\mathbf{E}_{\mathrm{f}} \cdot \mathbf{E}_{\mathrm{p}}^{*}$. The line shape reduces to a simple Lorentzian with a width twice the homogeneous width. In the presence of spectral diffusion, the hole excited by $\mathbf{E}_{\mathrm{f}} \cdot \mathbf{E}_{\mathrm{p}}^{*}$ diffuses in energy space resulting in a spectral redistribution of the excitation. Since tuning $\Omega_{\mathrm{b}}$ effectively changes $\Omega_{\mathrm{s}}$, this redistribution can be directly mapped out by the FWMb response. In this case, the FWMb response results from both the spectral hole burning and the spectrally diffused excitations.

It is important to note that while there are similarities between the FWMb line shape and the line shape obtained in ordinary spectral hole burning, there are two important differences. Hole burning basically measures the imaginary part of the optical response while the FWMb response measures the modulus square of the response, hence including both imaginary and real parts. More significantly, however, is that in frequency domain FWM, it is possible to control the amount of contributions from the spectrally diffused excitation by using a fixed forward pump and probe frequency offset as we can see from (5). In particular, in the limit of a large offset (much greater than the spontaneous emission rate), the FWMb response reflects only the homogeneous line shape. In practice, by choosing an appropriate offset, the contribution from spectrally diffused excitation may be eliminated without significant reduction of the nonlinear signal strength. Furthermore, while the current discussion has focused on a simple resonant system, it is not difficult to imagine that there are different systems characterized by different excitation and dephasing relaxation rates but having nearly the same resonant frequency. By examining the homogeneous line width measured in the FWMb response as a function of different forward pump and probe offsets, it is possible to differentiate between the different resonant systems [12].

Figure 2 shows two FWMb line shapes obtained from the nonlinear polarization in (5). Here, the strong redistribution kernel and the inhomogeneous distribution are taken to be Gaussian. The strong redistribution kernel is characterized by a center frequency $\omega_{0}$ and a half-width $\Delta \omega_{0}$. Similarly, the inhomogeneous distribution is characterized by a center frequency $\omega_{\text {inh }}$ and a half-width $\Delta \omega_{\text {inh }}$. The dashed line in the figure is the response obtained with $\Delta_{\mathrm{fp}}=0$. The narrow peak around zero detuning is predominantly the result of spectral hole burning while the broad peak reflects the spectral redistribution of the exci- 


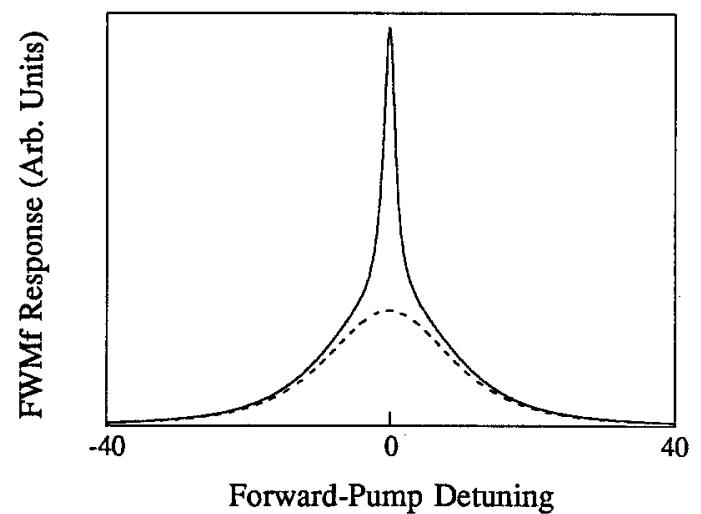

Fig. 3. The FWMb response. $\Gamma=15, \Delta \omega_{\text {inh }}=1000, \Delta \omega_{0}=400$, $\omega_{0}-\omega_{\text {inh }}=-100, \Omega_{\mathrm{p}}=\omega_{0}$. The response shows a prominent narrow component due to the decay of the quasi-equilibrium population. The width of this component is determined by the spontaneous emission rate. The dashed line represents the nonlinear response without contribution from the spectrally diffused excitations

tation created at the forward pump and probe frequency. The solid line in the figure represents the FWMb line shape using a forward pump and probe offset $\Delta_{\mathrm{fp}}=\Gamma$. The line shape is Lorentzian and reflects the homogeneous line shape of the system. Note that the strength of the nonlinear signal has only been reduced by a factor of order 2.

In the FWMf measurement, we hold $\Omega_{\mathrm{p}}=\Omega_{\mathrm{b}}$. Detuning the forward pump frequency by an amount $\delta=\Omega_{\mathrm{f}}-\Omega_{\mathrm{p}}$ produces a traveling wave modulation of excitation with the amplitude proportional to $[\delta+\mathrm{i}(\Gamma+$ $\left.\left.\gamma_{\mathrm{sp}}\right)\right]^{-1}$. The nonlinear response as a function of $\delta$ then measures the decay rate of the modulation formed by excitations that are resonant with the signal beam. The decay rate, $\Gamma_{\mathrm{m}}=\Gamma+\gamma_{\mathrm{sp}}$, includes contributions from spontaneous emission as well as transfer of excitations from energy $E$ to $E^{\prime}$ where $\left|E-E^{\prime}\right|>\Gamma_{\mathrm{h}}$. Spatial diffusion of the excitation, which is not included in (5), also contributes to the decay and manifests itself as a dependence of the decay rate on the spatial period of the modulation. Note that in the limiting case where $\Gamma_{\mathrm{h}} \sim \Gamma_{\mathrm{m}} / 2$, the FWMf line shape is complicated by the fact that tuning $\Omega_{\mathrm{f}}$ also changes the frequency of the signal beam and the frequency of the first order polarization. The FWMf response then experiences an additional resonant effect from the hole burning denominator appearing as $\left(\delta+2 \mathrm{i} \Gamma_{\mathrm{h}}\right)^{-1}$ [see (5)], resulting in a deviation from a simple Lorentzian and requiring a small correction (of order 1) in relating $\Gamma_{\mathrm{m}}$ to the HWHM for absolute decay rate measurements. The FWMp response provides a measurement similar to the FWMf response. However, since $\Omega_{\mathrm{f}}=\Omega_{\mathrm{b}}$, the hole burning denominator appears as $\left(\delta+\mathrm{i} \Gamma_{\mathrm{h}}\right)^{-1}$ resulting in a slightly larger correction as we can see from (5). In the case of $\Gamma_{\mathrm{h}} \gg \Gamma_{\mathrm{m}} / 2$, the FWMp and FWMf line shapes are the same and independent of $\Gamma_{\mathrm{h}}$.

Spectral diffusion of the excitation between different energy sites also leads to the establishment of a quasiequilibrium redistribution of the excited state population as we have discussed earlier. This is expected regardless

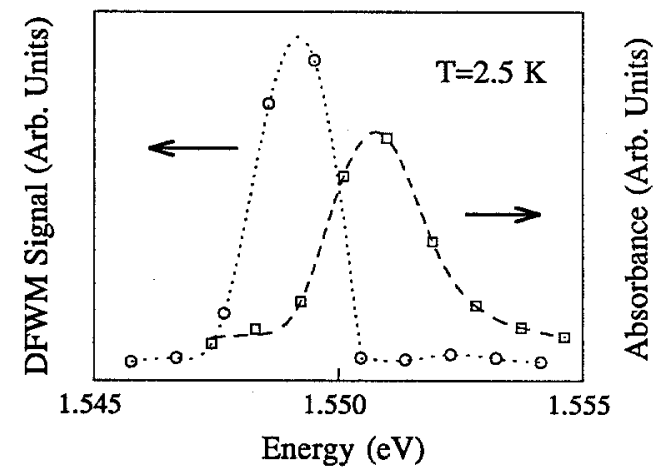

Fig. 4. The degenerate FWM (DFWM) and absorption spectra of $\mathrm{HH} 1$ excitons obtained at $2.5 \mathrm{~K}$

of the functional form of the redistribution kernel. This quasi-equilibrium population contributes to the nonlinear response, and decay of this population is determined by the spontaneous emission of the excited states. If the spontaneous emission rate is slow compared to the spectral diffusion rate, then a narrow resonance can be observed on top of the broad resonance with a half-width given by $\gamma_{\mathrm{sp}}$. In the limit of a very large spectral diffusion rate, the narrow resonance may even become dominant. In time resolved luminescence of this transition, the measured decay rate would be given by $\gamma_{\mathrm{sp}}$. However, because of the hole burning achieved in frequency domain FWM, it is possible to separately measure both decay rates. Figure 3 shows a FWMf line shape using the nonlinear polarization in (5). As anticipated, it clearly shows a prominent narrow peak sitting on top of a broad base. The broad base is the result of the fast spectral diffusion process $\left(\Gamma=15 \gamma_{\mathrm{sp}}\right)$. The narrow peak corresponds to the excited state spontaneous emission rate.

\section{High-Resolution Nonlinear Measurements}

Typical GaAs QW samples used in our measurements consisted of 65 periods of $96 \AA$ GaAs wells and $98 \AA$ $\mathrm{Al}_{0.3} \mathrm{Ga}_{0.7} \mathrm{As}$ barriers, grown at $630^{\circ} \mathrm{C}$ by a Varian Gen II MBE machine on semi-insulating (100) GaAs substrate with interrupted growth. The samples are mounted on a sapphire disk ( $c$ axis normal) with the substrate removed for the nonlinear measurement. The data presented here were obtained on a sample that is characterized by an absorption line width of $2.2 \mathrm{meV}$ for the HH1 exciton, and a Stokes shift of $1 \mathrm{meV}$ between the HH1 exciton absorption and emission. Similar results were also obtained on other samples. In contrast to many earlier measurements, the exciton density for these measurements were kept low near $10^{7}$ excitons $/ \mathrm{cm}^{2}$. All the nonlinear measurements are carried out on the HH1 exciton.

The excitonic nonlinear optical response is primarily due to phase space filling and exchange effects. Figure 4 shows the degenerate FWM response obtained at $2.5 \mathrm{~K}$. The rapid decrease of the nonlinear response around the absorption line center is partly due to strong exciton absorption. However, the nonlinear response on the high energy side of the absorption line center is extremely 

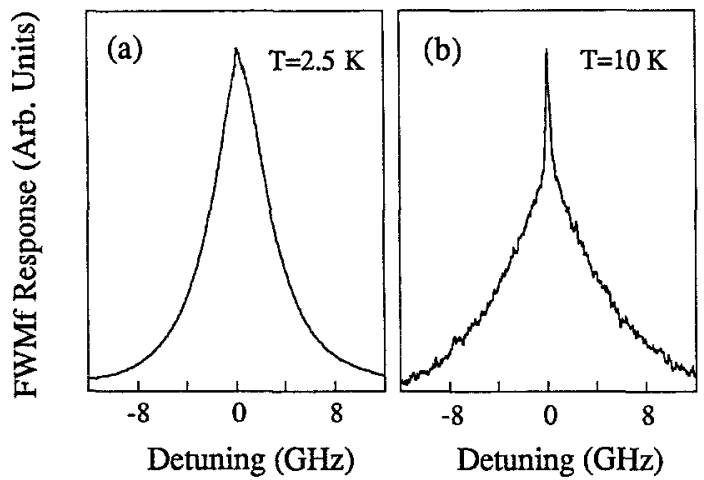

Fig. 5a, b. The FWMf response below the absorption line center. a At $2.5 \mathrm{~K}$ and at $1.5 \mathrm{meV}$ below the exciton absorption line center. b At $10 \mathrm{~K}$ and at $0.6 \mathrm{meV}$ below the exciton absorption line center. The line shapes show two decay components. The decay time associated with the line shape is $(2 \pi \cdot \mathrm{HWHM})^{-1}$. Compare with Fig. 3

small compared to that on the low energy side signaling a change of the exciton relaxation properties across the absorption line center. The small $\mathrm{cw}$ nonlinear optical response indicates a much increased exiton relaxation rate above the absorption line center. The energy dependence of the relaxation rate will be discussed in more detail later.

Relaxation of the exciton population is first characterized using the FWMf response [23]. A typical line shape below the absorption line center is shown in Fig. 5a. Complex decay dynamics of the exciton is evidenced in the line shape. The HWHM of the line shape corresponds to a relaxation time of $60 \mathrm{ps}$, which is slow compared to acoustic-phonon scattering of the delocalized exciton (typically on a time scale of $10 \mathrm{ps}$ ), and is over an order of magnitude faster than the exciton recombination time. Furthermore, within experimental error, the spectrum shown in Fig. 5a is independent of the modulation spacing, indicating the contribution from exciton diffusion is negligible and inferring an upper limit of order $1 \mathrm{~cm}^{2} / \mathrm{s}$ for the exciton diffusion coefficient. The above result indicates that excitons are strongly localized in this spectral region as suggested by earlier resonant Rayleigh scattering and transient FWM measurement of Hegarty and Sturge [9]. The measurements suggest that the decay of the exciton population is characterized by exciton spectral diffusion as a result of scattering of localized excitons from energy $E$ to $E^{\prime}\left(\left|E-E^{\prime}\right|>\Gamma_{\mathrm{h}}\right)$. The obtained decay rate is in agreement with the calculation based on phonon assisted migration of the localized exciton [6].

Excitons that have migrated out of sites with energy $E$ also have a finite probability to come back leading to the establishment of a quasi-equilibrium exciton population at energy $E$ before these ecitons eventually recombine, as discussed above. Decay of this quasi-equilibrium population should be characterized by the exciton recombination rate, and so is the nonlinear optical response due to this population. The FWMf response shown in Fig. 5a indeed shows a small and narrow feature at the top of the line shape. The narrow feature becomes more pronounced at higher temperature due to faster exiton

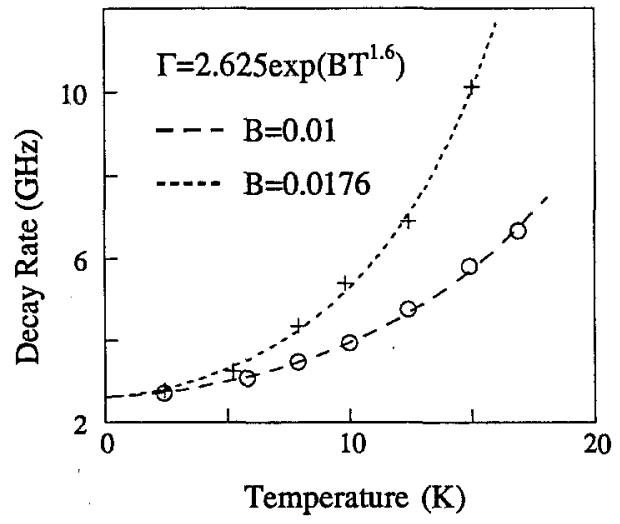

Fig. 6. The temperature dependence of the exciton population decay rate. Circles and crosses are data obtained at $1.5 \mathrm{meV}$ and at $0.6 \mathrm{meV}$ below the exciton absorption line center respectively. Dash lines are fit to the theory of phonon assisted migration

migration rate which also leads to an increase of the quasi-equilibrium population, as shown in Fig. 5 b. The width associated with the feature corresponds to a decay time of $1.2 \mathrm{~ns}$ consistent with the exciton recombination rate, which also confirms the above interpretation of the nonlinear measurements.

The mechanism of exciton migration can be best revealed by the temperature dependence of the migration rate since the migration process is likely to involve absorption or emission of acoustic phonons. The theoretical model for phonon assisted exciton mgiration was developed recently by Takagahara [6]. In this model, excitons resonantly excited are in a non-equilibrium state and can migrate to other sites by emitting or absorbing acoustic phonons. While the migration is due to the overlap of the exciton wave function in different sites when the inter-site distance is small, the inter-site dipole-dipole interaction mediates the migration process when the inter-site distance is much greater than the localization length. Typical magnitude of participating phonon wave vectors is within a few times of the inverse of the localization length corresponding to phonon energies of order 0.01 to $0.1 \mathrm{meV}$. The theory further predicts a distinctive temperature dependence for the migration rate. At low temperatures, the dependence is described by $\exp \left(B T^{\alpha}\right)$. In this expression, $B$ is positive and independent of temperature but is expected to increase with the exciton energy and depends on details of interface roughness; $\alpha$ is estimated to be between 1.6 and 1.7. The predicted temperature dependence is quite different from that of variable range hopping used by Mott to interpret electronic conduction in the localized regime. The difference has been attributed to the long-range nature of the inter-site interaction and the phonon emission process involved in the migration of the localized exciton [6]. The temperature dependence has been observed in transient hole burning experiments in an InGaAs/InP QW where all excitons are localized by alloy disorder [25].

Figure 6 shows the temperature dependence of the exciton migration rate obtained at $0.6 \mathrm{meV}$ and $1.5 \mathrm{meV}$ below the absorption line center using the FWMf re- 


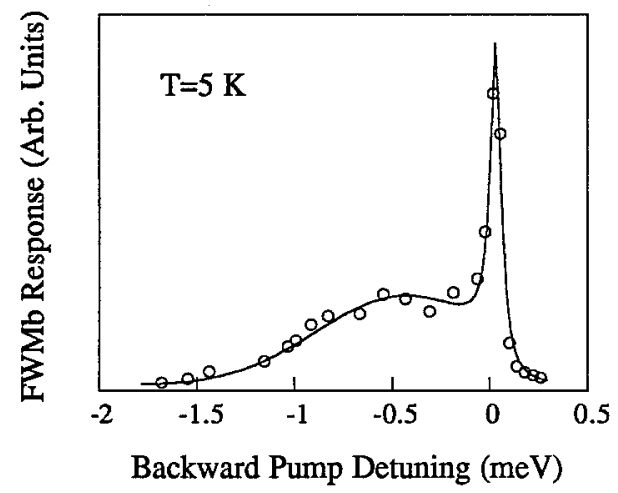

Fig. 7. The FWMb response at $5 \mathrm{~K}$ and with the frequencies of the forward pump and probe beams at $1.5 \mathrm{meV}$ below the exciton absorption line center. The narrow resonance is due to spectral hole burning. The response also provides a measure of the steady state spectral redistribution of the exciton population. Compare with Fig. 2

sponse. The data is in good agreement with the theory of phonon assisted migration discussed above with $\alpha=1.6$. The measurement indicates that the dominant contribution to relaxation of the localized exciton is phonon assisted migration up to a temperature of $15 \mathrm{~K}$. Note that earlier measurements have reported observations of an activation type of temperature dependence for the localized exciton at temperatures between 7 and $20 \mathrm{~K}$, indicating that in this temperature region, relaxation for the localized exciton is dominated by thermal activation to delocalized states $[8,10]$. It has been suggested that sample dependent variations in the thermal activation energy are the result of differences in the nature of interface roughness [26]. Hence, the effective activation energy can be much higher than simply the energy difference between the mobility edge and the localized exciton resulting, at low temperature $(<15 \mathrm{~K})$, in a thermal activation rate much smaller than the phonon assisted migration rate. Note that thermal activation type of behavior in our samples has been observed above $15 \mathrm{~K}$ using stimulated photon echo methods $[27,28]$.

A more complete description of exciton spectral diffusion can be achieved by making a direct measurement of the excitons scattered from energy $E$ to $E^{\prime}[24,29]$. If localized excitons are optically excited at energy $E$ and then migrate among localization sites to different energies, a quasi-equlibrium exciton distribution over a broad spectral range can be established assuming the exciton migration rate is large compared with the recombination rate. The steady state redistribution of the exciton population can be directly probed in the FWMb response by scanning $\Omega_{\mathrm{b}}$ while keeping $\Omega_{\mathrm{f}}$ and $\Omega_{\mathrm{p}}$ fixed at energy $E$, as anticipated in (5) and Fig. 2. Figure 7 shows a FWMb line shape where excitons are optically excited at $1.5 \mathrm{meV}$ below the absorption line center. The nonlinear response is corrected for sample absorption. The narrow resonance in the response corresponds to exciton spectral hole burning, and the width of the hole gives an exciton homogeneous width of order $\Gamma_{\mathrm{h}} \sim 0.01 \mathrm{meV}$. The broad feature is due to the quasi-equilibrium redistribution of the exciton population, and the square root of the response is proportional to the steady state exciton

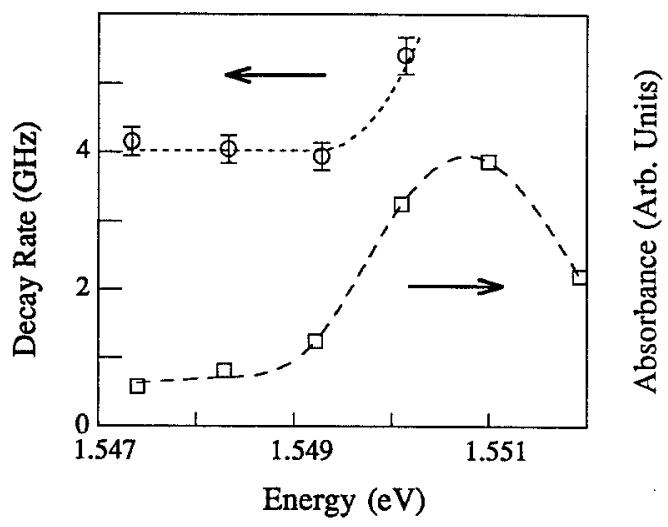

Fig. 8. The energy dependence of the exciton population decay rate at $10 \mathrm{~K}$. Dash lines are only a guide to the eye

population assuming all excitons in the spectral region concerned give rise to the same $\mathrm{cw}$ nonlinear response. The FWMb line shape in Fig. 7 can be fit to a simple model which neglects migration to states above the excitation energy, and is based on the understanding of the nonlinear optical response of a simple two-level system. The model assumes a Gaussian distribution for the quasiequilibrium population of excitons that have migrated to states below the excitation energy. The spectral profile of the hole burning resonance is assumed to be Lorentzian. The result is plotted as the solid line in Fig. 7.

We further examine the energy dependence of the exciton relaxation rate using the FWMf response. For exciton energies less than $1.5 \mathrm{meV}$ below the absorption line center, the exciton migration rate depends very weakly on the energy as is shown in Fig. 8. The migration rate increases rapidly approaching the absorption line center suggesting an increase of the localization length and an onset of the transition from localized to delocalized states. Physically, excitons can become delocalized when the localization length is comparable with or larger than the scale of inelastic cutoff given by $\sqrt{D \tau}$ [30], where $D$ is the exciton diffusion coefficient and $\tau$ is the time between exciton-phonon scattering.

Earlier measurements using transient four wave mixing and resonant Rayleigh scattering have suggested that, in a multiple QW, excitons above the exciton absorption line center may be weakly delocalized. Indeed, our measurements of FWMf and FWMb responses above the exciton absorption line center are qualitatively different from that below the absorption line center. The nonlinear response is completely dominated by the recombination component even at the lowest temperature $(1.8 \mathrm{~K})$ we can achieve. This behavior is expected from the earlier analysis of the FWMf response if excitons experience an extremely rapid inelastic scattering such as in the case of a delocalized exciton. Figure 9a shows a typical FWMf line shape above the exciton absorption line center. A large modulation spacing is used in this measurement so that contributions from exciton diffusion are negligible. The dashed line in the figure is a Lorentzian fit to the line shape. The FWHM of the line shape corresponds to an 

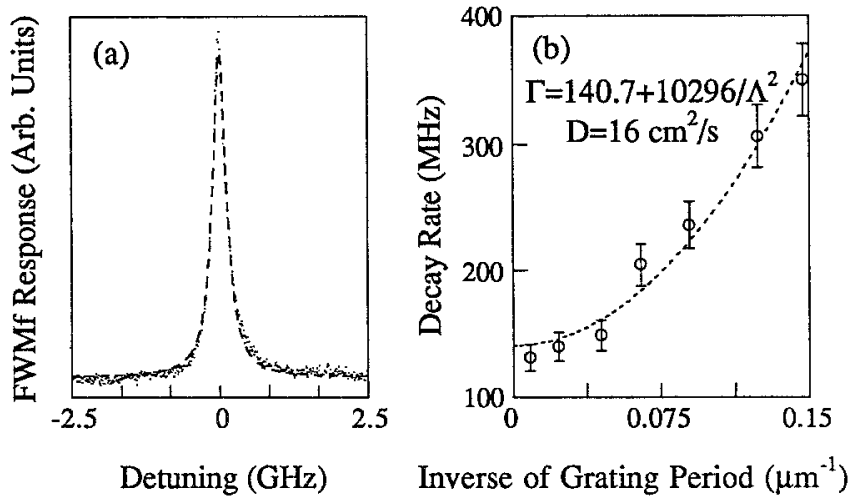

Fig. 9. a The FWMf response at $10 \mathrm{~K}$ and $2 \mathrm{meV}$ above the exciton absorption line center. The dashed line is a fit to the Lorentzian. b The dependence of decay rates of the exciton population modulation on the modulation spacing. The dashed line is a fit to the equation of modulation decay due to diffusion

exciton recombination time of $1.2 \mathrm{~ns}$. Furthermore, the measurement of the FWMf response as a function of the modulation spacing shows a quadratic dependence of the modulation decay rate on the inverse of the modulation spacing, yielding an exciton diffusion coefficient of order $16 \mathrm{~cm}^{2} / \mathrm{s}$ (see Fig. 9b).

The FWMb response from the delocalized excitons needs more clarification. As we have discussed earlier, in a FWMb measurement, tuning the frequency of the backward pump probes the steady state distribution of the exciton population. While delocalized excitons can be scattered along the two dimensional energy-momentum dispersion curve to other delocalized states by inelastic processes such as exciton-phonon interactions, these states have nonzero momentum, and in the limit of a strict $K$-selection rule, a zero optical dipole moment. In this case, the FWMb line shape in the vicinity of the excitation energy simply provides a measure of the exciton homogeneous line shape. Note that at very low temperature, delocalized excitons scattered to the bottom of the dispersion curve will continue to lose energy by emitting acoustic phonons and eventually become localized as we show below.

The FWMb line shape for excitons resonantly excited $2 \mathrm{meV}$ above the absorption line center is shown in Fig. 10. Narrow hole burning resonances are not observed in this line shape. The nonlinear signal above the absorption line center is extremely small compared to that below the line center, which is due to rapid dephasing of the polarization as well as the much increased exciton population decay rate. A magnified version of the FWMb spectrum above the absorption line center is also shown in the figure, from which we determine the homogeneous line width to be of order $1.5 \mathrm{meV}$ (corresponding to a dephasing time of $0.5 \mathrm{ps}$ ). The large dephasing rate for the delocalized exciton is expected $[6$, 9] since these excitons are expected to experience rapid elastic scattering from interface potential fluctuations in addition to the exciton-acoustic-phonon scattering. The strong nonlinear optical signal below the absorption line center indicates that most of the excitons created above

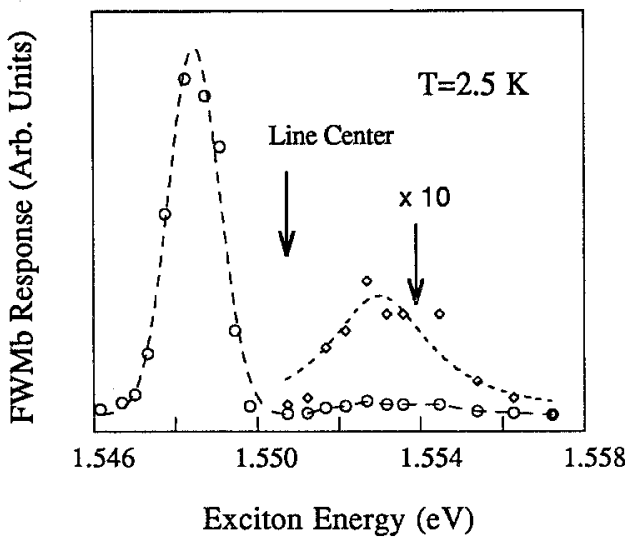

Fig. 10. The FWMb response at $2.5 \mathrm{~K}$ with the frequencies of the forward pump and probe beams at $2 \mathrm{meV}$ above the exciton absorption line center. Dashed lines are guide to the eye

the absorption line center become localized before they eventually recombine.

In summary, frequency domain nonlinear measurements have revealed features of various physical mechanisms of exciton relaxation in GaAs QW structures. In particular, phonon assisted migration has been found to dominate relaxation of the localized exciton at very low temperature. The high resolution measurements have also enabled us to directly determine the redistribution of the exciton population due to exciton migration. In addition, these measurements clearly demonstrated the effects of relaxation on frequency domain nonlinear optical response of the exciton.

Acknowledgements. This work has been supported by the U.S. Army Research Office and the Air Force Office of Scientific Research. MBE samples were provided by Prof. P.K. Bhattacharya.

\section{References}

1. See for example, S. Schmitt-Rink, D.S. Chemla, D.A.B. Miller: Adv. Phys. 38, 89 (1989)

2. S. Schmitt-Rink, D.S. Chemla, D.A.B. Miller: Phys. Rev. B 32, $6601(1985)$

3. See for example, H. Sakaki, T. Noda, K. Hirakawa, M. Tanaka, M. Matsusue: Appl. Phys. Lett. 51, 1934 (1987)

R. Gottinger, A. Gold, G. Abstreiter, G. Weiman, W.Schlapp: Europhys. Lett. 6, 183 (1988)

A. Ourmazd, D.W. Taylor, J. Cunningham, C.W. Tu: Phys. Rev. Lett. 62, 933 (1989)

4. See for example, C.W. Tu, R.C. Miller, B.A. Wilson, P.M. Petroff, T.D. Harris, R.F. Kopf, S.K. Sputz, M.G. Lamont: J. Cryst. Growth 81, 159 (1987)

5. See for example, M. Tanaka, H.Sakaki: J. Cryst. Growth 81, 153 (1987)

6. T. Takagahara: Phys. Rev. B 31, 6552 (1985); B 32, 7013 (1985)

7. Y. Masumoto, S. Shionoya, H. Kawaguchi: Phys. Rev. B 29, 2324 (1984)

8. J. Hegarty, L. Goldner, M.D. Sturge: Phys. Rev. B30, 7346 (1984)

9. J. Hegarty, M.D. Sturge: J. Opt. Soc. Am. B 2, 1143 (1985)

10. J.E. Zucker, A. Pinczuk, D.S. Chemla, A.C. Gossard: Phys. Rev. B 35, 2829 (1987)

11. T. Yajima, H. Souma: Phys. Rev. A 17, 309 (1978) 
12. J.T. Remillard, H. Wang, D.G. Steel, J. Oh, J. Pamulapati, P.K. Bhattacharya: Phys. Rev. Lett. 62, 2861 (1989)

13. See for example, Optical Phase Conjugation, ed. by R.A. Fisher (Academic,New York 1983)

14. For $\mathrm{cw}$ measurements, the orientational grating $\left(\mathbf{E}_{\mathrm{p}} \perp \mathbf{E}_{\mathrm{f}} \| \mathbf{E}_{\mathrm{b}}\right)$ has not been observed, and hence there is no back grating due to $\mathbf{E}_{\mathrm{b}} \cdot \mathbf{E}_{\mathrm{p}}^{*}$ if $\mathbf{E}_{\mathrm{p}} \perp \mathbf{E}_{\mathrm{b}}$

15. H. Wang, D.G. Steel: Phys. Rev. A 43, 3823 (1991)

16. P.R. Berman: Phys. Rep. 43, 102 (1978)

17. T.F. Soules, C.B. Duke: Phys. Rev. B 3, 262 (1971)

18. W.E. Lamb, Jr.: Phys. Rev. A 143, 1429 (1964)

19. J.R. Klauder, P.W. Anderson: Phys. Rev. 125, 912 (1962)

20. M. Lindberg, S.W. Koch: Phys. Rev. B 38, 3342 (1988)

21. P.R. Berman, R.G. Brewer: Phys. Rev. A 32, 2784 (1985)
22. D.G. Steel, J.T. Remillard: Phys. Rev. A 36, 4330 (1987)

23. I.D. Abella, N.A. Kurnit, S.R. Hartmann: Phys. Rev. 141, 391 (1965); see also T. Yajima, Y. Taira: J. Phys. Soc. Japan 47, 1620 (1979)

24. H. Wang, M. Jiang, D.G. Steel: Phys. Rev. Lett. 65, 1255 (1990)

25. J. Hegarty, K. Tai, W.T. Tsang: Phys. Rev. B 38, 7843 (1988)

26. T. Takagahara: Private communication

27. M.D. Webb, S.T. Cundiff, D.G. Steel: Phys. Rev. Lett. 66, 934 (1991)

28. M.D. Webb, S.T. Cundiff, D.G. Steel: Phys. Rev. B 43, 12658 (1991)

29. H. Wang, J.T. Remillard, M.D. Webb, D.G. Steel, J. Pamulapati, J. Oh, P.K. Bhattacharya: Surf. Sci. 228, 69 (1990)

30. D.J. Thouless: Phys. Rev. Lett. 39, 1167 (1977) 